\title{
A Comprehensive Analysis of QoS in Wired and Wireless SDN Based on Mobile IP
}

\author{
Nazrul Islam \\ Department of Information and Communication Technology, Mawlana Bhashani Science and Technology University, \\ Tangail-1902, Bangladesh \\ E-mail: nazrul.islam@ieee.org
}

\section{Md. Habibur Rahman*}

Department of Information and Communication Technology, Bangabandhu Sheikh Mujibur Rahman Digital University, Bangladesh

*Corresponding Author E-mail: habibur@ict.bdu.ac.bd

\section{Mostofa Kamal Nasir}

Department of Computer Science and Engineering, Mawlana Bhashani Science and Technology University, Tangail1902, Bangladesh

E-mail: kamal.mostofa@gmail.com

Received: 27 March 2021; Accepted: 07 June 2021; Published: 08 October 2021

\begin{abstract}
Software-Defined Networking (SDN) is the new network paradigm whose primary focus is to create a dynamic, scalable and flexible network. It provides programmable functions for implementation of network configuration management. The demand for wireless network functionality is rising simultaneously. Mobility management for a large network is an issue in Internet Engineering Task Force (IETF). There are also a number of solutions to support mobility within the network. The Mobile Internet Protocol (MIP) is used for managing mobility. Furthermore, for the SDN platform, mobility adds roaming capability for mobile nodes in the software-defined wireless network (SDWN). In the wired scenario, SDN has different capabilities to deliver network services according to the fixed node. This study provides a quality of service (QoS) analysis in both SDN and SDWN. Mininet WiFi and RYU network emulator as a controller is used to implement the mobility API. The random walk model is applied as mobility functionality toward the final nodes. Moreover, several QoS measurement matrices are analyzed according to the network topology. At the end, round trip time (RTT), cumulative distributed function (CDF), packet loss and throughput are analyzed for quality of service comparable in the SDN and SDWN scenarios according to the MIP.
\end{abstract}

Index Terms: QoS, SDN, SDWN, Micro-Mobility, Mininet Wi-Fi, Mobile IP.

\section{Introduction}

The most emergent network architecture that provides dynamicity, adaptability and cost-effectiveness are referred to as Software Defined Network (SDN). It is used on complex and heterogeneous networks and large commercial aspects. The SDN enables programming in the network design. The control plane is decoupled from forwarding plane $[1,2]$. All routing decisions are monitored through centralized devices called controllers. Decision management is carried out through the network APIs. The SouthBound API was accustomed to talking with redirection devices like network switches in both directions [3]. OpenFlow protocols manage a controller's routing decision to change the flowhold rules of the flow table in switches $[4,5]$.

Network applications using "NorthBound" APIs are placed onto the controllers [6]. It maintains communication and delivers different on-demand network services programmed applications. Figure 1 illustrates the core SDN architecture. This SDN analogy is similar to how applications are programmed into computer operating systems This API is written inside the network operating system (NOS) of the controllers. This approach provides ease of node mobility. The mobility development of the Internet, making the mapping function programmable for packet flows [7]. The architecture of SDN enhances the distributing functionality. This centralizes the mobility management for distributed Home Agent [8]. Under the Software Defined Networking (SDN) scenario, the data transfer network components are connected directly to the central SDN controller. The device is connected to a physical transmission medium. The SDN is most appropriate for centralized one-controller access to all network entities in a small geographical area. 
Software Defined Wireless Network (SDWN) is the complete SDN solution for Wireless-Personal Area Networks [9]. SDWN supports flexible policy-making for SDN controllers. The mobile nodes can run legacy protocol stack for granted backward and peer compatibility on vast and complex geographical expansion [9]. The quality of service (QoS) of such a SDN made up of mobile nodes is currently required. Random node mobility requires a comparative perspective appropriate to SDWN in order to build a flexible, scalable and heterogeneous wireless network. The QoS of the underlying SDN network is defined by the settings, throughput, Round Trip Time (RTT), packet in message processing and Packet Loss Ratio, etc. [10]. Additionally, Software Defined Wireless Network (SDWN) offers programmability and centralized control outside of Wireless Access Points (PA). SDWN has got emerging research importance soon after the increased attention of mobile network operator on this technology [11, 12].

Previous studies performed on SDWN include OpenRoad proposals to improve robustness during mobility handoff using OpenFlow network [13]. On paper [14], Cell SDN is proposed for LTE network-based operating system for the cellular wireless network. According to study [15], the function of distributed mapping is added for Mobility Management. For mobility management, both wireless and mobile networks have gotten the researchers' attention where WLAN got most works paid off $[13,16]$. No works are done before of SDN for both mobile nodes and use of middleboxes.

This study was about implementing middleboxes in the SDN for mobile nodes. This study presents a qualitative analysis of the quality of service for the wired SDN and SDWN. The research work is carried out by analyzing QoS measures on SDN where nodes have mobility. Parallel analysis of SDWN mobile nodes is performed. To disguise the mobility solution, Mobile IP (MIP) takes into consideration in this simulation illustration. The system uses a Care-ofAddress (CoA) to route to a new network. To accomplish the objective of this research, the Mininet-WiFi network emulator is used to create and observe QoS measurements for the two Mobile IP-based simulation scenarios. With the emergence of more and more heterogeneous wireless and complex networks, the current situation is legitimate for conducting research on the SDWN and the corresponding QoS. The real-time oriented sector adapting the SDN architecture into the broader wireless scenario will have to correctly study the knowledge base and measurements.

However, to our knowledge, there is no comparative analysis of the SDN and the SDWN is done according to the performance parameters of the network. In addition, a first step is to implement SDN for mobile nodes and middlebox uses. Secondly, an analysis of the performance of the affected network for the SDN and SDWN is necessary. A comprehensive quality of service survey is complementary for further deployment of the network.

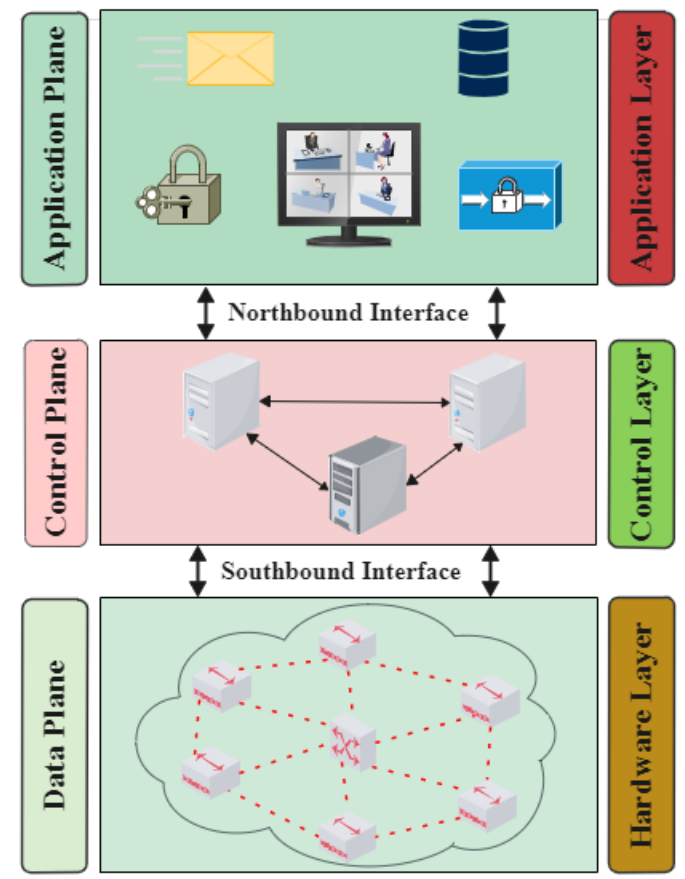

Fig.1. Architecture of Software Defined Network

The rest of the document is organized in the following way. Section 2, contains the context and associated work for Wireless SDN and Mobility Management. Section 3 presents the methodology used to carry out the study. Section 4 outlines the topology design and implementation of the proposed design to analyze QoS parameters. Section 5 presents the findings and analysis of the work and Section 6 concludes the conclusion with other research perspectives. 


\section{Background and Related Work}

Mobility in the wireless network means the change of point of attachment and the IP address for a Mobile Node (MN). Hence, mobility management of conventional SDN and SDWN is crucial to assume a comprehensive advantage of their perspective deployment. Various research and proposition of several SDN based systems are performed for effective mobility management [17].

Handling the mobility of the wireless network is the difficult task. The mobility architecture of the SDWN is illustrated in [18]. Mobile IP functionality is clearly described in the paper [19]. Research findings in the paper [16] demonstrated and evaluated the mobility of an SDN based IP network. Triangle routing occurs while maintaining the host's mobility in an SDN network. The programmable control plane is used to solve triangle routing. While updating the routing table of the network switches, the control plane controls the routing information. The Middlebox provides the fast-routing facilities. The use of middleboxes, provides higher throughput, as well as lower latency, dramatically improving Quality of Service (QoS) [20]. The essential idea of this study was implementing the middle-box for mapping functionality to multiple locations in the network. In paper [21], a solution for IP based Mobility Support in SDNs evaluated for multiple inter-domain communication. However, mobility maintenance in the SDWN becomes the essential simulation.

Later on, with the emergency use of the wireless network, several further researches conducted in Software Defined Wireless Network (SDWN). Mao et al. [17] interpreted these two technologies, SDWN and Mobile Wireless Network (MWN). The researchers investigate their joint diverse heterogeneous network design with crucial challenge and significant future benefits in a wireless network. They represent the successful implementation of two converged network technologies. It improved the resource utilization, customized services, innovation from the network layer to the physical layer and moreover guaranteed QoS. Monitoring the performance of QoS in the SDN the application "MonSamp" is successfully implemented [22]. The highest quality of service for the business customer in SDN is achieved in the Study [23]. However, no one has studied the comparative analysis of SDN and SDWN with Mobile IP.

Hence, the above outcome and endless possibilities of SDN in mobility network are analyzed. This paper represents the QoS analysis and comparative performance study on SDN architectures, both in the wired and wireless scenario. This paper provides the mobility of the hosts in the network architecture on its control plane. However, the paper also implements the middle box for better outcomes of Quality of Service (QoS) on its simulation.

\section{Research Method}

A comprehensive analysis is performed in RYU controller based SDN network architecture in both wired and wireless scenarios. The performance based on Quality of Service (QoS) and anomaly detection of such network architecture has been evaluated in VPN protocol and fuzzy logic [24, 25, 26]. This study compares the SDN data plane's performance of QoS along with the mobility management. The mobility functions that are used for the mobile nodes are based on the Random Direction and Random Walk Model [27]. Several network simulation tools are used to analyze such type of network simulation including Mininet Wi-Fi [28], OMNET++ [29], Network Simulator-2 (NS-2) [30], Network Simulator-3 (NS-3) [31], EstiNet [32], OpenNet [33]. Mininet Wi-Fi has been chosen to allow the programmability on the control plane of SDN network using python language. However, Mininet Wi-Fi has the advance experimental benefits for both updated APIs and mobile hosts allowing research on SDWN. It is used for the network simulation and packet generation for further evaluation. The simulation has been performed in a virtual machine using VirtualBox with the configuration- Intel's Core-i5 processor with 4 GB memory.

This study provides a comprehensive analysis of the wired and wireless SDN in terms of QoS parameters. Python (v 3.6.0) programming is used to configure both the control plane and data plan of the network. In terms of evaluating the performance of mobility management of the network 53 data sets are generated and captured by Wireshark in both wired and wireless SDN [34]. At the end of each simulation, final evaluation has been considered by averaging the result of performance metrics of QoS. A comparative analysis has been presented to conclude the study.

\section{Design and Implementation}

The SDN network topology has been designed to analyze the QoS performance while mobility is present in both wired and wireless scenarios. Fig. 2 and Fig. 3 depicts the network connectivity of the components and the functionality of the wired and wireless SDN network respectively. The topology is designed with a single RYU controller, four network domains (A, B, C and D). Mobile nodes are connected with network access points (APs) while corresponding nodes are connected by modem. Open Flow (v 1.4) protocol is used as a standard communication protocol [35]. RYU controllers manage the network while the mobile nodes follow the random walk model for their mobility function [36].

The mobility controller RYU is connected with the mobility management application through northbound API. The router and access points (APs) are connected to the RYU controller via southbound and northbound API respectively. For the mobility handover session, two correspondence nodes (CN-1 and $\mathrm{CN}-2)$ and two mobile nodes 
(MN-1 and MN-2) are used. For the continuous flow connection, the access points are connected via a secure channel. The simulation mechanism is described in the bellow sub-section.

\subsection{Implementing Wired SDN}

Flow entry in wired SDN is recorded for each new position of the mobile nodes. The MN-1 connects to the AP in the network domain A (Fig. 2). At the time of communicating with the correspondence node (CN-1) and MN-1 moves, the access point sends an acknowledgment to the RYU controller to hand over the session. A short lifetime of the entry is managed to minimize the total number of flows for all nodes in the controller. Before running the simulation of the wired SDN topology in Mininet-WiFi, the flow entry is pre-downloaded into the controller to ensure the reachability of each node in the network. The performance of the topology is measured running Iperf command in the terminal between the communicating nodes.

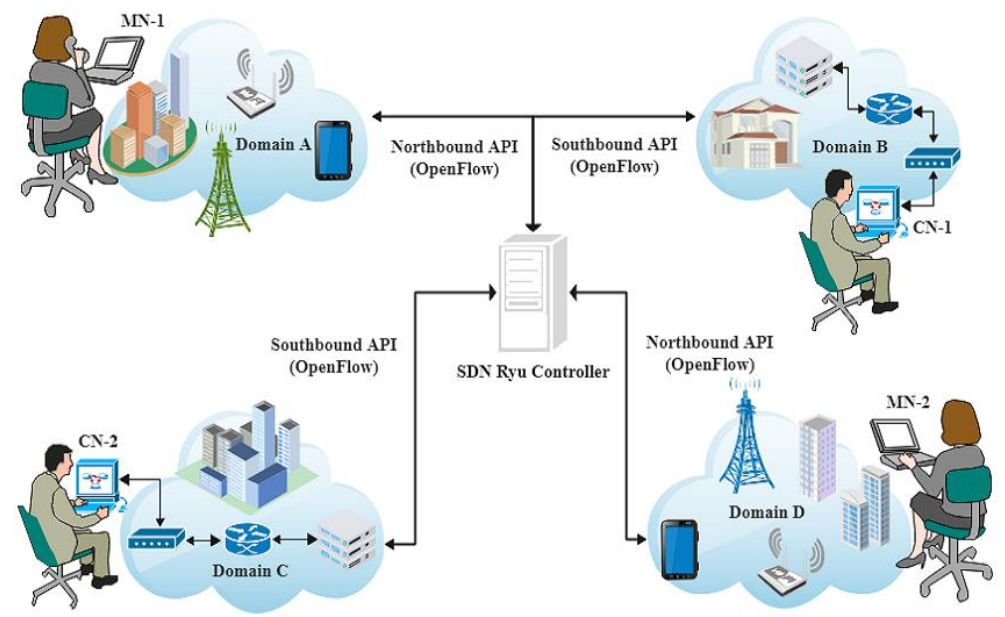

Fig.2. Wired SDN network topology with fixed correspondence node.

\subsection{Implementing Wireless SDN}

The Mininet WiFi emulator is used for SDWN context like as SDN. The nodes that connect the APs through WLAN interface is shown in Fig. 3. In this case, a bridge is created among the OpenFlow switch and WiFi interface. When both the correspondence nodes $(\mathrm{CN}-1)$ and $\mathrm{MN}-2$ are moved from one place to another in the same access point, the flow entity is handed over. Considering the domain B and D in Fig. 3, the MN-2 is connected in the AP in domain D. When it moved towards the domain $\mathrm{B}$, it sends an acknowledgment to the controller to hand over the session. The RYU controller smartly handles the session and provides the continuous flow connection.

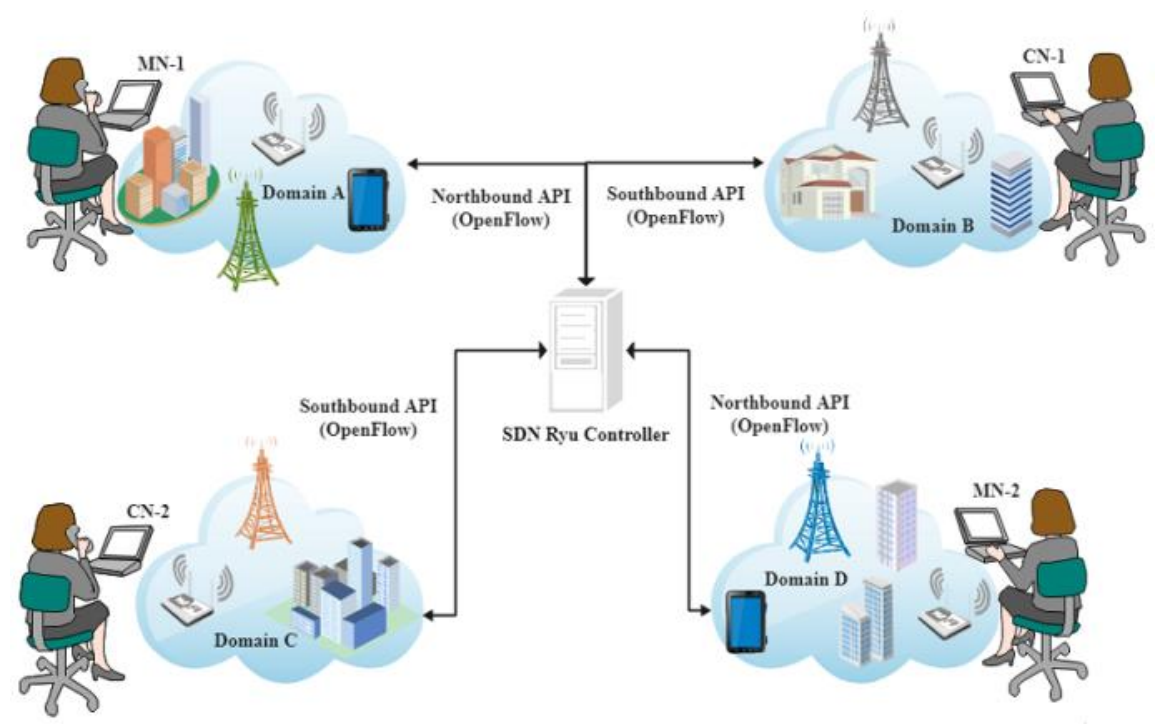

Fig.3. Wireless SDN network topology with mobile correspondent node. 


\subsection{Mobile IP based Mobility Management}

Consistent data connectivity is essential while changing the point of attachment of a mobile node in a network. This feature is enabled by mobility management protocols that ensure the reachability of the mobile terminal. Mobile IPv4 (MIPv4), Mobile IPv6 (MIPv6), Fast handover for MIPv6 (FMIPv6), Hierarchical MIPv6 (HMIPv6) are widely used as mobility management protocols. Therefore, numerous number of network design is developed in order to get the best use of the mobility management procedure. The overview of the MIP mobility model is displayed in Fig. 4.

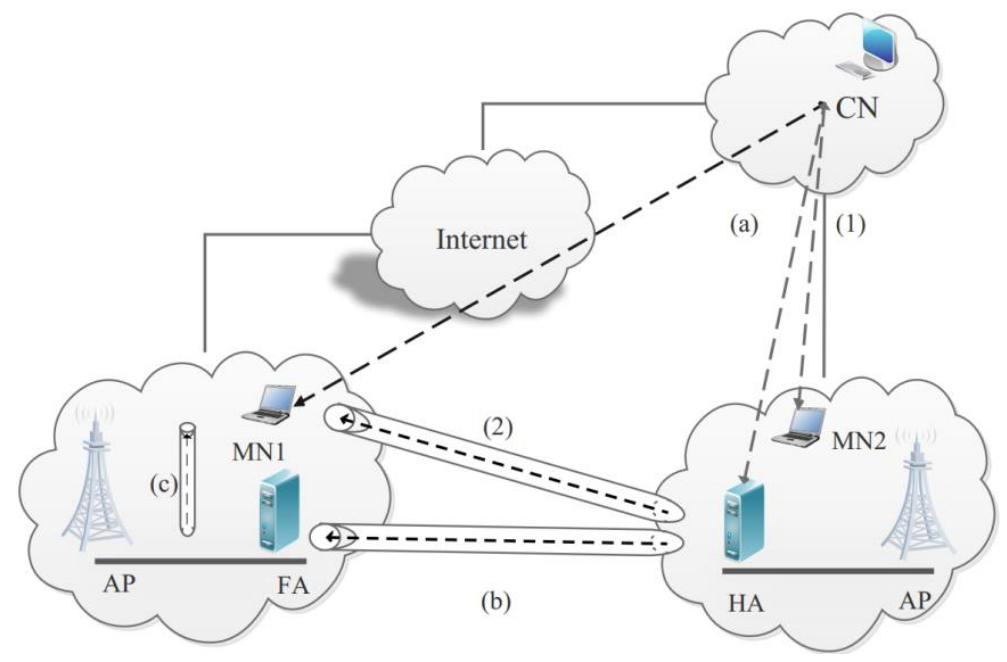

Fig.4. Basic terminology of typical MIP based mobility model

When the MN1 stays in its home network the MNs packets are routed in a standard way from the MNs HA towards the MN directly using the MNs HoA address. When the MN2 moves away from its home network to a visited network, which is covered by the FA, the MN begins the Agent Discovery phase, which detects its movement in the new network using Agent Solicitation/Advertisement messages towards FA. Then, the Registration phase takes place when the MN discovers its location in a foreign network and obtains a new CoA. This coat can be acquired using Soliciting/Advertisement messages or through query-response operation towards Dynamic Host Configuration Protocol (DHCP) [10]. Then, the MN updates the mobility binding in its HA with this new CoA in order to associate this CoA with its HoA address. Lastly, in the data transfer phase, all MN packets sent from the MNs CN towards MN are intercepted by the MNs HA. When the HA receives any packets destined for a particular MN it will encapsulate these packets and tunnels them to the MNs CoA through the serving FA using tunnel ends at the FA, which will de-capsulate these packets and forward them to the MN, as shown in steps (a), (b) and (c) in Fig. 4.

For the different mobility management methods described before it can be possible to explore common procedures deployed internally in the SDN controller to be applied to all the cases, facilitating the interworking of them, and complemented by the specific functional modules per method. Furthermore, it can be completed by moving the remaining particularities to the edge in the case of the user plane aspects.

Furthermore, in both wired and wireless SDN scenarios, packet interval is set 2 seconds and transmitted packet numbers are 100 for each simulation. After simulating the network, the traffic is captured in the Wireshark. The packet details are exported in the comma separated values (CSV) file. Then the captured results are plotted on the graph using MATLAB. The performance metrics of QoS including TCP sequences, round trip time (RTT), throughput, packet loss and cumulative distributed function (CDF) are represented in the result analysis section.

\section{Result Analysis}

This section illustrates a comparative study and analysis of QoS parameters in both wired SDN and wireless SDN architecture using the Mininet WiFi emulator. In the topological design, the corresponding nodes are fixed in the wired connection. On the other hand, in wireless SDN, the mobile nodes (MN) functions, mobility in getting detached from the current Access Point (AP) and therefore attached to the nearby one. Corresponding node (CN) updates the flow table based on each new entry forwarded by the switch or APs resultant from mobility functionalities. This mobility model with Mininet WiFi follows Random Way Point (RWP) to move around the MNs in the testbed. MN in the SDWN testbed connects the strongest signal providing in nearby AP and leaves the previously connected AP.

The implementation follows with two distinguished scenarios with similar topological architecture. The first topology includes basic LAN based wired network performs on OpenFlow and MIP. The second inherits from wireless nodes MN, connected with the SDN controller via switching devices called wireless APs. In both cases, the controller is considered as dynamic and scalable with network size, hence RYU controller has been in use. MIP model redirects all 
the correspondent node $(\mathrm{CN})$ to the mobile node $(\mathrm{MN})$ traffic once the $\mathrm{MN}$ moves to a new switching AP. The traffic handover is performed on the strongest signal first basis.

Two sets of analysis are performed based on Mininet WiFi testbed implementation. The first set analyzes the throughput, round trip time and packet loss ratio for obtaining OpenFlow and TCP packet transmission to represent the QoS in both wired SDN and wireless SDN topologies. The later set includes the control plane overhead of both the topologies to represent scalability in each scenario.

\subsection{TCP Sequence}

Time sequence graph refers to the graphical presentation of collecting TCP sequence the simulation time span. For the above-mentioned network along with TCP sequence there will be OpenFlow packets. Hence, the captured packets will hold the communication between AP-MN and Controller-AP. During handover, based on the transition smoothness, the peak of the sequence will be disrupted and recovered as the sinlessness of the network.

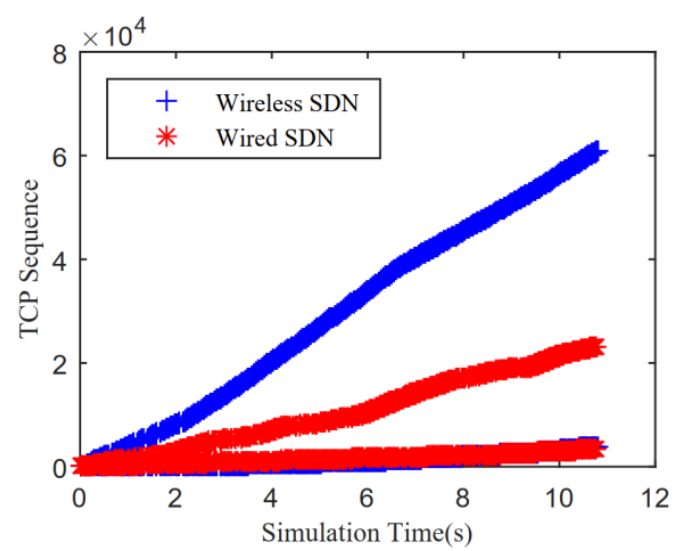

Fig.5. TCP sequence with respect to time for wired and wireless SDN during handoffs

Collected TCP time sequence of this handoffs in both wired and wireless SDN is depicted graphically in Fig. 5. This illustration indicates that degraded handoff efficiency in wired SDN where retransmission caused some packet loss keeping some space of sequences in between. A movement among the subnets also caused timeouts. The simulation is performed for 10 seconds for appropriate analysis in both scenarios. In both scenarios, sequences are captured twice, once during controller-AP communication and another AP-MN communication. It depicts for all cases, the controllerAP sequence is horizontal and throughput is still. On another hand, throughput increases with time from the wireless network than wired SDN. MIP is creating efficient handoff for SDWN inheriting more throughputs over time. Wired SDN finds, it's time to consume to reach the mark because of complexity in handling efficiency on IP based mobility management scenario.

\subsection{Round Trip Time (RTT)}

Round trip transmission time graph represents the graphical situation of raise and decrease in RTT for the packets over simulation time. The RTT value of all the above-mentioned protocols temporarily can rise to a higher value during handoffs. The higher the value, the less efficient the handover of the mobility handover of the network. Besides, with the SDN based network with OpenFlow, RTT decreases with next handover every time.

The RTT was originally estimated by.

$$
R T T=\alpha+(1-\alpha) * \text { PreviousRTT }
$$

Where: $\alpha=$ Constant weighted factor $(0 \leq \alpha<1)$

With the equal simulation time Fig. 6 shows the RTT in wired and wireless network testbed collected from the Mininet WiFi testbed. The Y-axis exhibit RTT (s) and the X-axis present simulation time (s). The red and blue dots represent RTT samples calculated for non-retransmitted segments in wired and wireless SDN. It is vigilant that, RTT levels in both cases rise higher during handoffs. However, wired SDN stays with a higher RTT value than wireless SDN requires for each handoff. This is because wireless SDN avoids triangle routing choosing the strongest signal providing switches for MIP ensuring optimal forwarding path. Besides, RTT value of wireless SDN increase with handoff number. 


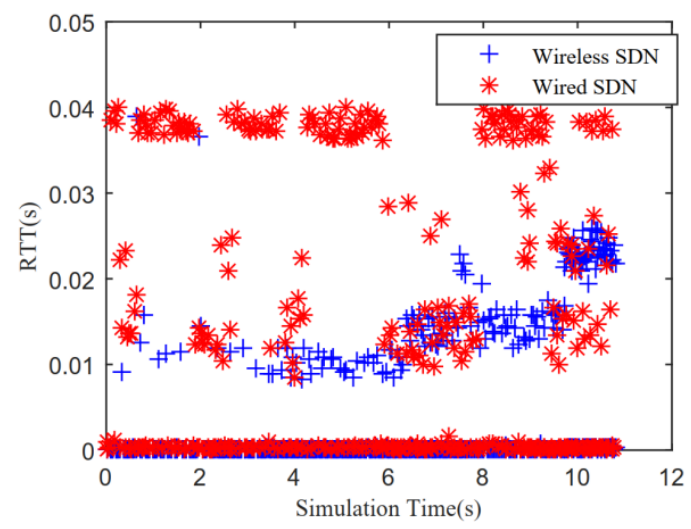

Fig.6. RTT values of simulation events during handoff situations

\subsection{Throughput and Packet Loss}

Throughput graph refers to the presentation of successful packet transmission between the nodes in a given time [37]. Any point of that representation denotes the throughput of that particular moment for that packet communication. It has been calculated by bellowing formula.

$$
\begin{gathered}
\text { Throughput }=\frac{\text { Packet Size }}{\text { Round Trip Time }} \\
\text { PER }=\frac{\text { Error Packet }}{\text { Tested packet }} * 100 \%
\end{gathered}
$$

Packet Error Rate (PER) has been calculated based on the confidence level of more than eighty successful packet transmission. Controls whether the confidence level will be used during the PER measurement. If the confidence level is on, the PER measurement will continue until a pass or fail indication occurs, or until the maximum packet count is reached. If the confidence level is off, the PER measurement will run until the maximum packet count is reached. The required confidence level for the PER requirement (range is $80 \%$ to $99.99 \%$ ). The PER percentage that must not be exceeded for a pass result. To be returned (range is $0.1 \%$ to $15 \%$ ). The ratio of the packet error count of the number of packets tested in per cent $(\%)$ is termed as packet error rate.

Throughput and packet loss ratio has also been analyzed for both wired and wireless SDN network. With different mobility frequency of $0,0.5$ and 1 per sec. Iperf is performed between $\mathrm{CN}-1$ and $\mathrm{MN}-1$ about 10 times taking 1 min each time. This simulation depicts Fig. 7 illustrating higher average throughput in each mobility frequency for SDWN compared with wired SDN. The results also represent in Fig. 8 that, SDWN has 40\% lower UDP packet loss compared with wired SDN because of handoff efficiency and MIP mobility solution in wireless SDN.

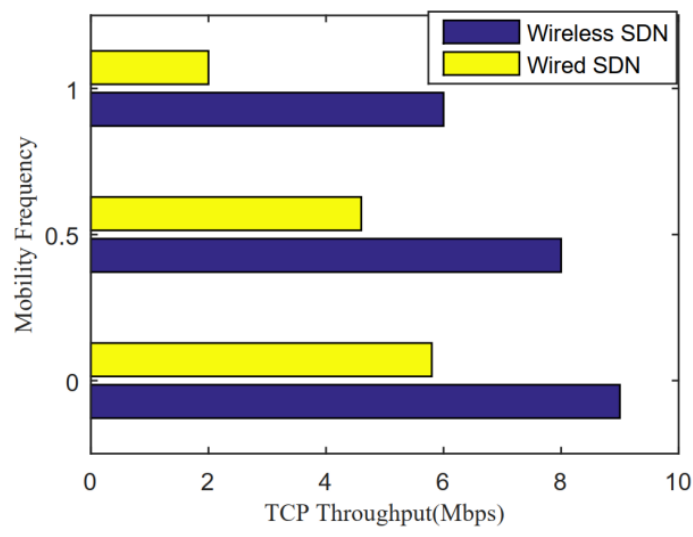

Fig.7. TCP throughput of wired and wireless SDN with varied mobility frequency 


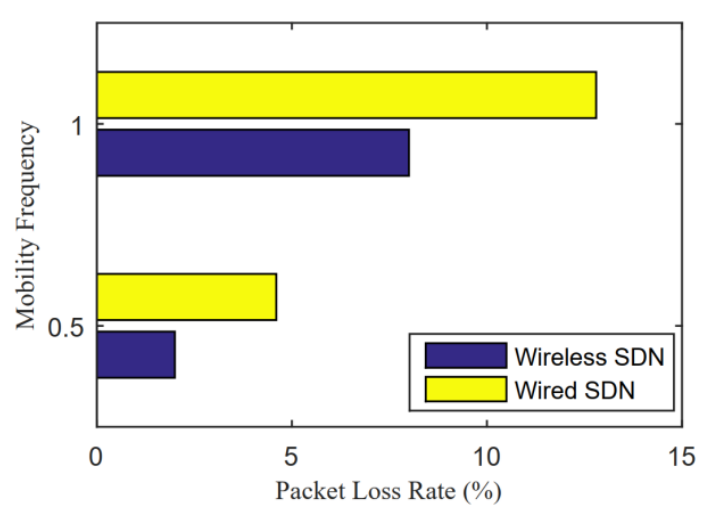

Fig.8. UDP packet loss ratio of wired and wireless SDN with varied mobility frequency

\subsection{Cumulative Distribution Function (CDF)}

The distribution of the input parameters over some certain values holds the probabilistic distribution which calls the cumulative distribution function (CDF). This function, if represented in graphical manner will hold the probability of the input that holds any value between the given value ranges. Any point of the graph holds the probability of the $\mathrm{X}$ value in $\mathrm{X}$-axis to have the probability of referring $\mathrm{Y}$ in the $\mathrm{y}$-axis. This research considers the mobility frequency as the range for maximum and average packet in messages in the controller for transmission.

In simulation data, the maximum packet-in messages per second turned 35 and average packet-in messages are 20s got both the scenarios for the simulation duration. These values represent the overhead and possible scalability of the network. Later cumulative distributed function $(\mathrm{CDF})$ of average and maximum packet-in messages are calculated. The CDF of average and maximum packet-in messages in handling session handovers by the controller in both wired and wireless SDN is depicted in Fig. 9 and Fig.10 respectively. The figure depicts similar outcome in simulation results, about 10 to 13 messages are received by the controller in each scenario with distinguished inter domain movement probability $\mathrm{p}(0.01,0.1,0.75)$. This increased CDF in wireless SDN illustrates more scalability than the scalability in wired SDN.

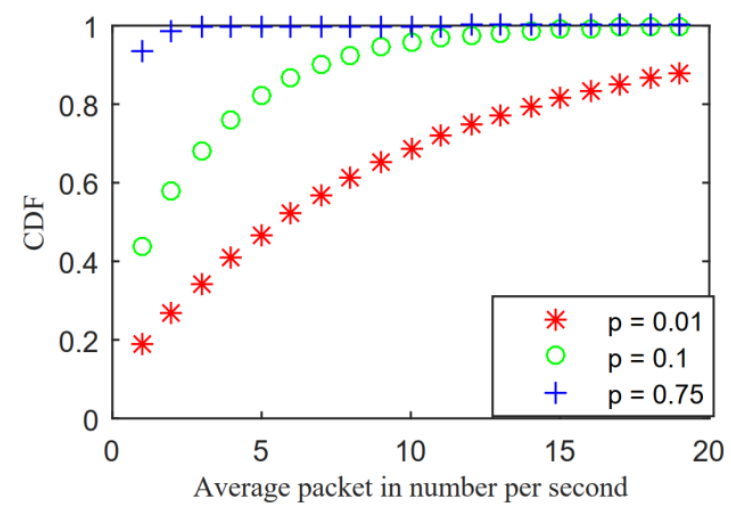

Fig.9. Cumulative distribution function of average packet-in messages

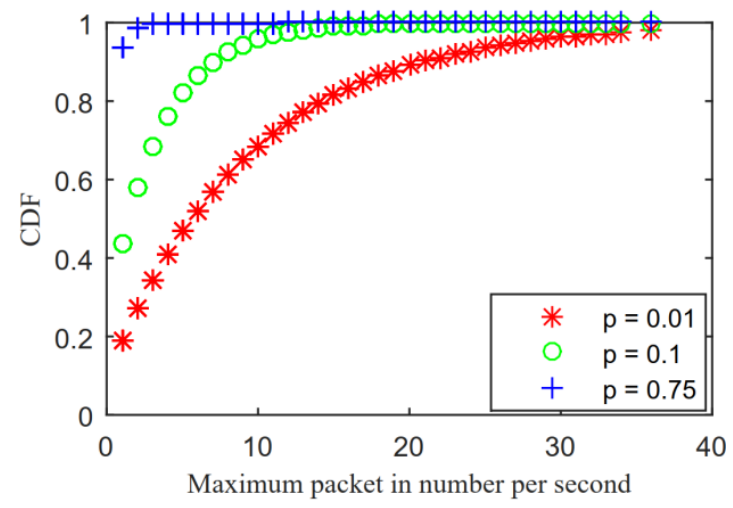

Fig.10. Cumulative distribution function of maximum packet-in messages 
The results demonstrate that the probability of inter domain movement slightly affects average and maximum packet-in messages. In conclusion, largest overhead is the cause of burst packet-in messages which is not prevalent here The larger the network, the higher the number of packets. More packets transmit through the controller and its efficiency decreases. Fig. 9 and Fig. 10 also depict less scalability options for wired SDN network than SDWN. Hence, the network compared to wired SDN, SDWN demonstrates scalable performance in packet overhead management.

\section{Conclusions}

The present article is a study of QoS analysis on wired and wireless SDN. It concludes a presentation of comparative analysis in parallel with an integration of IP mobility management. The results of the Mininet Wi-Fi test bed of these two types of networks showed a visible state in terms of QoS settings. The findings show that the wired SDN has a significantly lower TCP rate, a higher UDP packet loss ratio than the value of the wireless SDN. The higher transmission time during transfers is vigilant in the wire line SDN relative to the wireless SDN QoS measurements. The reason for this difference is the efficient transfer, the MIP mobility solution for wireless SDN and wired SDN is delayed in transfer efficiency. The MIP mobility model is less time consuming, less retransmission time in the wireless SDN. But both networks demonstrate the feasibility and scalability of controller performance. Consequently, the study concludes that wireless SDN in a complex cellular network is more adaptable.

In the future, prototyping the network in a real-time environment will be beneficial. The security outlook on the transport layer of SDWN will be a research potential for a complex cellular network.

\section{References}

[1] Rahman, M. H., Islam, N., Swapna, A. I., \& Habib, M. A. (2020, February). "Analysis of Software Defined Wireless Network with IP Mobility in Multiple Controllers Domain.” In International Conference on Cyber Security and Computer Science (pp. 529-538). Springer, Cham.

[2] Abdolhossein Fathi, Keihaneh Kia,"A Centralized Controller as an Approach in Designing NoC", International Journal of Modern Education and Computer Science (IJMECS), Vol.9, No.1, pp.60-67, 2017.DOI: 10.5815/ijmecs.2017.01.07

[3] Lo, C. C., Wu, P. Y., \& Kuo, Y. H. (2015, November). "Flow entry conflict detection scheme for software-defined network." In 2015 International Telecommunication Networks and Applications Conference (ITNAC) (pp. 220-225). IEEE.

[4] Jany, M. H. R., Islam, N., Khondoker, R., \& Habib, M. A. (2017, December). "Performance analysis of OpenFlow based software defined wired and wireless network." In 2017 20th International Conference of Computer and Information Technology (ICCIT) (pp. 1-6). IEEE.

[5] Eric Gamess, Daniel Tovar, Alberto Cavadia, "Design and Implementation of a Benchmarking Tool for OpenFlow Controllers", International Journal of Information Technology and Computer Science (IJITCS), Vol.10, No.11, pp.1-13, 2018. DOI: 10.5815/ijitcs.2018.11.01

[6] Shivayogimath, C. N., \& Reddy, N. U. (2016). "Performance analysis of a software defined network using Mininet." In Artificial Intelligence and Evolutionary Computations in Engineering Systems (pp. 391-398). Springer, New Delhi.

[7] Valtulina, L., Karimzadeh, M., Karagiannis, G., Heijenk, G., \& Pras, A. (2014, December). "Performance evaluation of a SDN/OpenFlow-based Distributed Mobility Management (DMM) approach in virtualized LTE systems." In 2014 IEEE Globecom Workshops (GC Wkshps) (pp. 18-23). IEEE.

[8] Chan, M. C., Chen, C., Huang, J. X., Kuo, T., Yen, L. H., \& Tseng, C. C. (2014, April). "OpenNet: A simulator for softwaredefined wireless local area network.” In 2014 IEEE Wireless Communications and Networking Conference (WCNC) (pp. 3332-3336). IEEE.

[9] Costanzo, S., Galluccio, L., Morabito, G., \& Palazzo, S. (2015, September). "Software defined wireless network (SDWN): An evolvable architecture for W-PANs." In 2015 IEEE 1st International Forum on Research and Technologies for Society and Industry Leveraging a better tomorrow (RTSI) (pp. 23-28). IEEE.

[10] Perkins, C. (2002). IP mobility support for IPv4.

[11] Bernardos, C. J., De La Oliva, A., Serrano, P., Banchs, A., Contreras, L. M., Jin, H., \& Zúñiga, J. C. (2014). "An architecture for software defined wireless networking." IEEE wireless communications, 21(3), 52-61.

[12] Sama, M. R., Contreras, L. M., Kaippallimalil, J., Akiyoshi, I., Qian, H., \& Ni, H. (2015). "Software-defined control of the virtualized mobile packet core." IEEE Communications Magazine, 53(2), 107-115.

[13] Yap, K. K., Huang, T. Y., Kobayashi, M., Chan, M., Sherwood, R., Parulkar, G., \& McKeown, N. (2009). "Lossless Handover with n-casting between WiFi-WiMAX on OpenRoads." ACM Mobicom (Demo), 12(3), 40-52.

[14] Li, L. E., Mao, Z. M., \& Rexford, J. (2012, October). "Toward software-defined cellular networks." In 2012 European workshop on software defined networking (pp. 7-12). IEEE.

[15] Chan, H. A., Yokota, H., Xie, J., Seite, P., \& Liu, D. (2011). "Distributed and dynamic mobility management in mobile internet: current approaches and issues." JCM, 6(1), 4-15.

[16] Wang, Y., Bi, J., \& Zhang, K. (2015). "Design and implementation of a software-defined mobility architecture for IP networks." Mobile Networks and Applications, 20(1), 40-52.

[17] Yang, M., Li, Y., Jin, D., Zeng, L., \& Wu, X. \&amp; Vasilakos, AV (2015). "Software-defined and virtualized future mobile and wireless networks: A survey." Mobile Networks and Applications, 20(1).

[18] Jin, X. (2006). A Survey on Network Architectures for Mobility.

[19] Akyildz, I. X. J., Mohanty, S. (2004), "A survey of mobility management in next-generation all IP-based wireless systems". IEEE Wireless Communications. v11 i4. pp16-27. 
[20] Tu, R., Wang, X., Zhao, J., Yang, Y., Shi, L., \& Wolf, T. (2015, April). "Design of a load-balancing middlebox based on SDN for data centers." In 2015 IEEE Conference on Computer Communications Workshops (INFOCOM WKSHPS) (pp. 480-485). IEEE.

[21] Wang, Y., \& Bi, J. (2014, August). “A solution for IP mobility support in software defined networks.” In 2014 23rd International Conference on Computer Communication and Networks (ICCCN) (pp. 1-8). IEEE.

[22] Raumer, D., Schwaighofer, L., \& Carle, G. (2014, September). "Monsamp: A distributed sdn application for QoS monitoring." In 2014 Federated Conference on Computer Science and Information Systems (pp. 961-968). IEEE.

[23] Sharma, S., Staessens, D., Colle, D., Palma, D., Goncalves, J., Pickavet, M., ... \& Demeester, P. (2014, April). Demonstrating resilient quality of service in Software Defined Networking. In 2014 IEEE Conference on Computer Communications Workshops (INFOCOM WKSHPS) (pp. 133-134). IEEE.

[24] Narayan, S., Williams, C. J., Hart, D. K., \& Qualtrough, M. W. (2015, January). "Network performance comparison of VPN protocols on wired and wireless networks." In 2015 International Conference on Computer Communication and Informatics (ICCCI) (pp. 1-7). IEEE.

[25] Anju Bhandari, V.P. Singh,"Design of Fuzzy-Based Traffic Provisioning in Software Defined Network", International Journal of Information Technology and Computer Science (IJTCS), Vol.8, No.9, pp.49-61, 2016. DOI: 10.5815/ijitcs.2016.09.07

[26] Gagandeep Garg, Roopali Garg,"Accurate Anomaly Detection using Adaptive Monitoring and Fast Switching in SDN", International Journal of Information Technology and Computer Science (IJITCS), vol.7, no.11, pp.34-42, 2015. DOI: 10.5815/ijitcs.2015.11.05

[27] Bettstetter, C., Hartenstein, H., \& Pérez-Costa, X. (2004). "Stochastic properties of the random waypoint mobility model." Wireless Networks, 10(5), 555-567.

[28] Fontes, R. R., Afzal, S., Brito, S. H., Santos, M. A., \& Rothenberg, C. E. (2015, November). "Mininet-WiFi: Emulating software-defined wireless networks." In 2015 11th International Conference on Network and Service Management (CNSM) (pp. 384-389). IEEE.

[29] Varga, A. (2010). “OMNeT++.” In Modeling and tools for network simulation (pp. 35-59). Springer, Berlin, Heidelberg.

[30] Teerawat, I., \& Hossain, E. (2009). "Introduction to network simulator NS2.” In Springer (pp. 1-18).

[31] Henderson, T. R., Lacage, M., Riley, G. F., Dowell, C., \& Kopena, J. (2008). "Network simulations with the ns-3 simulator." SIGCOMM demonstration, 14(14), 527.

[32] Wang, S. Y., Chou, C. L., \& Yang, C. M. (2013). "EstiNet OpenFlow network simulator and emulator.” IEEE Communications Magazine, 51(9), 110-117.

[33] Chan, M. C., Chen, C., Huang, J. X., Kuo, T., Yen, L. H., \& Tseng, C. C. (2014, April). "OpenNet: A simulator for softwaredefined wireless local area network." In 2014 IEEE Wireless Communications and Networking Conference (WCNC) (pp. 3332-3336). IEEE.

[34] Orebaugh, A., Ramirez, G., \& Beale, J. (2006). "Wireshark \& Ethereal network protocol analyzer toolkit.” Elsevier.

[35] Hossen, M. S., Rahman, M. H., Al-Mustanjid, M., Nobin, M. A. S., \& Habib, M. A. (2019, December). "Enhancing Quality of Service in SDN based on Multi-path Routing Optimization with DFS.” In 2019 International Conference on Sustainable Technologies for Industry 4.0 (STI) (pp. 1-5). IEEE.

[36] Islam, M. T., Islam, N., \& Al Refat, M. (2020). "Node to node performance evaluation through RYU SDN controller." Wireless Personal Communications, 1-16.

[37] Mahmood Z. Abdullah, Nasir A. Al-awad, Fatima W. Hussein, " Evaluating and Comparing the Performance of Using Multiple Controllers in Software Defined Networks", International Journal of Modern Education and Computer Science (IJMECS), Vol.11, No.8, pp. 27-34, 2019.DOI: 10.5815/ijmecs.2019.08.03.

\section{Authors' Profiles}

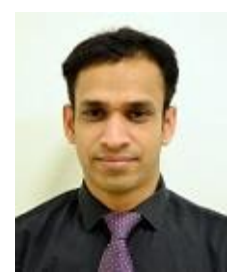

Nazrul Islam holds a Master of Science degree in Electrical Engineering with an emphasis on Telecommunication Systems from Blekinge Institute of Technology (BTH), Karlskrona, Sweden in January 2014. $\mathrm{He}$ also graduated from the Department of Information and Communication Technology (ICT) of Mawlana Bhashani Science and Technology University, Tangail-1902, Bangladesh in June 2008. He secured 2nd position in merit list of the batch. However, currently, he is working as an Assistant Professor, Department of Information and Communication Technology (ICT) at the Mawlana Bhashani Science and Technology University, Tangail1902, Bangladesh. He also worked as a Project Assistant for European Project-Quality of Experience Estimators in Networks (QUEEN). His research interests include Communication Networks (QoE), Software Defined Network (SDN), Machine Learning for Optical Sensing.

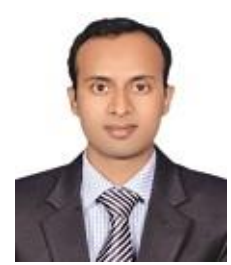

Md. Habibur Rahman completed his B.Sc. (Engg.) and M.Sc. (Engg.) from the Department of Information and Communication Technology at Mawlana Bhashani Science and Technology University, Tangail-1902, Bangladesh. He has received Prime Minister Glod Medal-2017 award from honorable Prime Minister Sheikh Hasina, Government of the People's Republic of Bangladesh organized by University Grants Commission of Bangladesh. His research is focused on the Software Defined Wireless Network (SDWN), IoT, Bioinformatics, and Biomedical Image Processing. Rahman is an active IEEE member since 2016. Currently he is serving as a Lecturer at the Department of Information and Communication Technology, Bangabandhu Sheikh Mujibur Rahman Digital University, Bangladesh. 


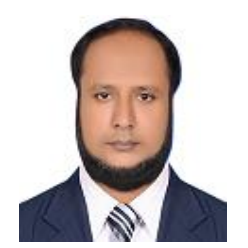

Mostofa Kamal Nasir Professor of Computer Science and Engineering at Mawlana Bhashani Science and Technology University, Tangail, Bangladesh. He has completed his PhD from University of Malaya, Kuala Lumpur, Malaysia in the field of Mobile Adhoc Technology in 2016. Before that he has completed his BSc and MSc in Computer Science and Engineering from Jahangirnagar University, Bangladesh. His current research interest includes VANET, IoT, SDN and WSN.

How to cite this paper: Nazrul Islam, Md. Habibur Rahman, Mostofa Kamal Nasir, "A Comprehensive Analysis of QoS in Wired and Wireless SDN Based on Mobile IP", International Journal of Computer Network and Information Security(IJCNIS), Vol.13, No.5, pp.18-28, 2021. DOI: 10.5815/ijcnis.2021.05.02 